\title{
Relationship between epicardial adipose tissue thickness and coronary thrombus burden in patients with ST-elevation myocardial infarction
}

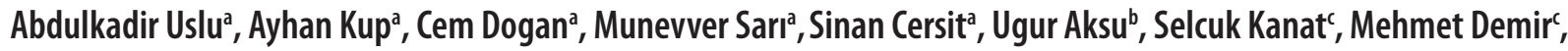 \\ Erhan Tenekecioglu ${ }^{c, d}$
}

Background. Epicardial fat reflects abdominal visceral adiposity and visceral fat plays an important role in the development of an unfavorable metabolic and atherosclerosis risk profile. Intracoronary thrombus burden is an important factor affecting the success of the procedure particularly in patients undergoing percutaneous coronary intervention $(\mathrm{PCl})$. Therefore, determining the factors predicting thrombus burden has great importance in predicting adverse cardiovascular events as well as determining the most appropriate treatment strategy to prevent failure in $\mathrm{PCl}$.

Aim. The aim of the current study was to evaluate the relationship between Epicardial adipose thickness (EAT) and thrombus burden in the patients with ST-elevation myocardial infarction (STEMI) who undergo primary PCI (pPCI).

Methods. The study was prospective and included patients $(n=156)$ who were referred to Kosuyolu Research and Education hospital with STEMI between 2016 and 2017. Thrombus burden was scored as follows: 0 (no thrombus), 1 (possible thrombus), 2 (definite thrombus $<0.5$ xreference vessel diameter), 3 (definite thrombus $0.5-2 x$ reference vessel diameter), 4 (definite thrombus $>2 x$ reference vessel diameter), and 5 (complete vessel occlusion). According to thrombus grade the patients were grouped as low thrombus burden (grades 0-3) and high thrombus burden (grades 4 and 5). EAT, identified as an echo-free space between the myocardium and visceral pericardium, was measured perpendicularly, on the free wall of the right ventricle at both parasternal long- and short-axis views at end-diastole in three cardiac cycles.

Results. Fifty-one subjects were in the low thrombus burden group and 105 in the high thrombus burden group. There were no differences in the two groups for LVEF, smoking status, family history of coronary artery disease (CAD), diabetes mellitus (DM), hypertension (HT), and hypercholesterolemia and for total cholesterol, triglyceride, GFR, LDL-C and HDL-C. In multivariate logistic regression analysis the EAT (odds ratio: $2.53,95 \% \mathrm{Cl}: 1.76-3.67 ; \mathrm{p}<.001$ ) was found as an independent predictor of high thrombus burden.

Conclusions. The present study showed that EAT was an independent predictor of coronary thrombus burden in STEMI.

Key words: thrombus burden, epicardial adipose thickness, acute coronary syndrome

Received: December 20, 2018; Revised: April 4, 2019; Accepted: August 12, 2019; Available online: September 16, 2019 https://doi.org/10.5507/bp.2019.038

(c) 2020 The Authors; https://creativecommons.org/licenses/by/4.0/

${ }^{a}$ Department of Cardiology, Kartal Kosuyolu Heart Research and Training Hospital, Istanbul, Turkey

${ }^{b}$ Department of Cardiology, Erzurum Education and Research Hospital, Erzurum, Turkey

'Department of Cardiology, Bursa Education and Research Hospital, Health Sciences University, Bursa, Turkey

${ }^{d}$ Department of Cardiology, Thorax Center, Erasmus MC, Erasmus University, Rotterdam, the Netherlands

Corresponding author: Erhan Tenekecioglu, e-mail:drercardio2@gmail.com

\section{INTRODUCTION}

Atherosclerotic plaque rupture induced coronary thrombus and the interruption of coronary blood flow are the main mechanisms initiating acute coronary syndromes (ACS) (ref. $\left.{ }^{1}\right)$. Coronary thrombus burden is one of the risk factors for long and short term adverse cardiovascular events in ACS (ref. ${ }^{2}$ ). Thrombus burden has impact on the success of the percutaneous coronary intervention (PCI) in ACS ( ref. $^{3}$ ). Even several invasive and pharmacological treatment modalities have been introduced, intracoronary thrombus management is still insufficient. Therefore, determining the factors in prediction of intracoronary thrombus burden has great importance in forecasting adverse cardiovascular events as well as determining the most appropriate treatment strategy to prevent any failure in PCI.

Epicardial adipose tissue (EAT) is a true visceral fat depot localized beneath the visceral pericardium. It has become evident that EAT is a metabolically active organ producing proinflammatory and proatherogenic cytokines $^{4}$. Increased EAT was associated with coronary no-reflow, coronary artery disease, hypertension (HT), insulin resistance and metabolic syndrome ${ }^{5}$. Given the potential paracrine effects of EAT, its distance from the epicardial coronaries and myocardial interstitium may be important factors in the development of myocardial microvascular dysfunction and thrombus formation ${ }^{4}$.

Previously, it was reported that inflammation was independently related to both no-reflow phenomenon and 


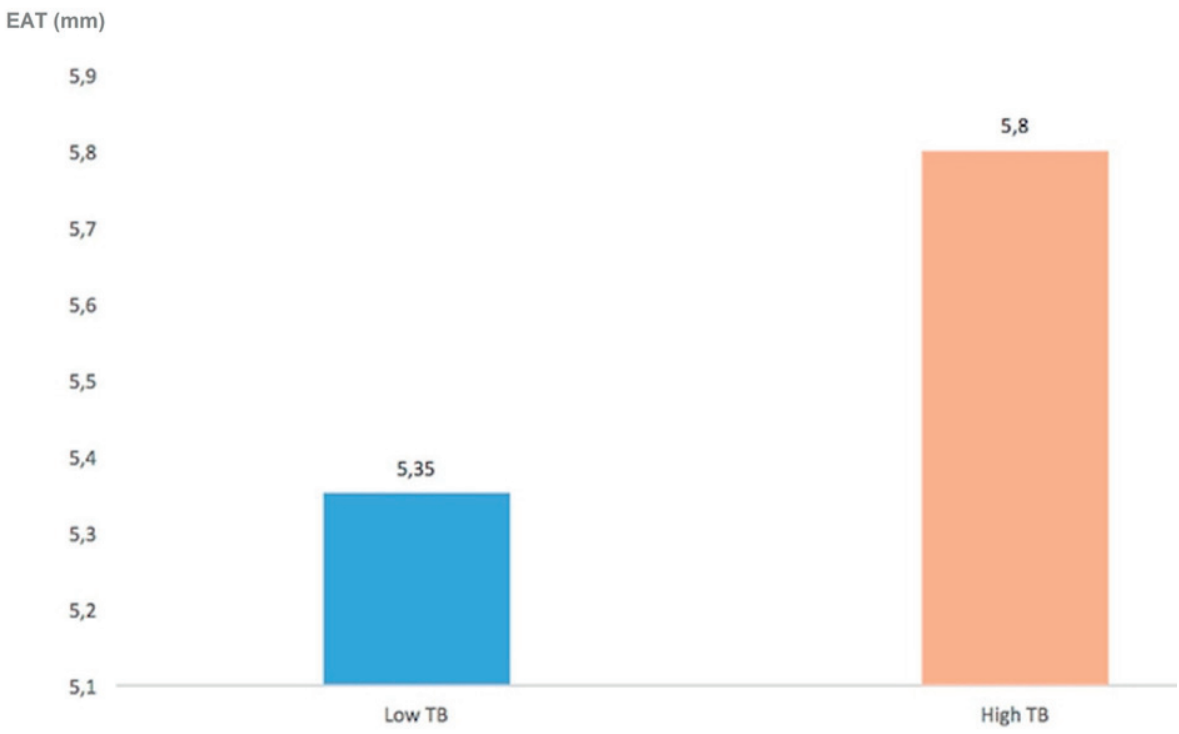

Fig. 1. The EAT value between low vs high thrombus burden.

the coronary thrombus formation in ACS (ref. ${ }^{6}$ ). The aim of this study was to evaluate the relationship between EAT thickness and thrombus burden in patients with STEMI who undergo primary PCI (pPCI).

\section{METHODS}

\section{Study Population}

The study recruited 156 patients prospectively. Patients who were referred to Kosuyolu Research and Education hospital with STEMI between 2016 and 2017 were enrolled into the study. The inclusion criteria were (1) presentation within the first $12 \mathrm{~h}$ after the onset of chest pain (18 hours for cardiogenic shock) and no history of thrombolytic therapy and (2) presence of an STsegment elevation at least $1 \mathrm{~mm}(2 \mathrm{~mm}$ for V1-V3) in 2 or more contiguous leads in electrocardiography (ECG). The patients who underwent thrombolytic therapy due to STEMI within $24 \mathrm{~h}$, cardiogenic shock on admission, had active infection, pre-diagnosed systemic inflammatory disease history, liver disease, renal failure were excluded. The study protocol was approved by the institutional review board in our center and informed consent was obtained from all of the patients.

The following data were recorded during the hospitalization; detailed medical history, complete physical examination, cardiovascular risk factors including age, sex, family history of coronary artery disease (CAD), current smoking status, HT, dyslipidemia, and diabetes mellitus (DM). Furthermore, the body mass index (BMI; weight $[\mathrm{kg}] /$ height squared $\left.\left[\mathrm{m}^{2}\right]\right)$ glomerular filtration rate (GFR) and hematological indices, lipid profile, creatine kinase (CK), creatine kinase-MB isoform (CK-MB) and troponin I levels were measured. The GFR was calculated from serum creatinine using the Cockcroft-Gault equation $([140-$ age $] \times$ [weight in $\mathrm{kg}] \times[0.85$ if female $] /$ $[72 \times$ creatinine $])$.

\section{Echocardiographic analysis}

Transthoracic echocardiography was performed in the left lateral decubitus position using a Vivid S5 system (GE Vingmed Ultrasound AS, Norten, Norway) with a $2.5 \mathrm{MHz}$ phased-array transducer. Transthoracic echocardiographic examinations were done by an experienced echocardiographer blinded to the study protocol according to the recommendations of the American Society of Echocardiography ${ }^{7}$. Left ventricular ejection fraction (LVEF) was determined using modified Simpson's method $^{8}$. EAT, identified as an echo-free space between the myocardium and visceral pericardium ,was measured perpendicularly, on the free wall of the right ventricle from both parasternal long-axis and short-axis views at end diastole in three cardiac cycles ${ }^{9}$. The maximum values at each site were quantified and the average value was used for the statistical analysis.

\section{Angiographic analysis}

Following the diagnosis of STEMI, the patients underwent emergency coronary angiography. All patients were administered as loading dose of $300 \mathrm{mg}$ of acetylsalicylicacid and clopidogrel $(600 \mathrm{mg})$ before the coronary intervention. Intravenous bolus of heparin $(100 \mathrm{U} / \mathrm{kg})$ was given to all cases and platelet GP IIb/IIIa inhibitors (tirofiban) were given in the catheterization laboratory at the discretion of the operator during coronary angiography. pPCI was performed using the standard femoral approach with a 7-Fr guiding catheter. Coronary angiograms were recorded in right and left oblique planes using cranial and caudal angulations, at a rate of 30 frames/s. During coronary angiography, iopromide (Ultravist 370, Schering AG, Berlin, Germany) was used as the contrast agent in all patients. After these standard procedures, angiographic thrombus burden was scored in five grades: 0 (no thrombus), 1 (possible thrombus), 2 (definite thrombus $<0.5 \mathrm{x}$ reference vessel diameter), 3 (definite thrombus 0.5-2 x reference vessel diameters), 4 (definite thrombus $>2 \mathrm{x}$ reference vessel diameters), and 5 (complete 


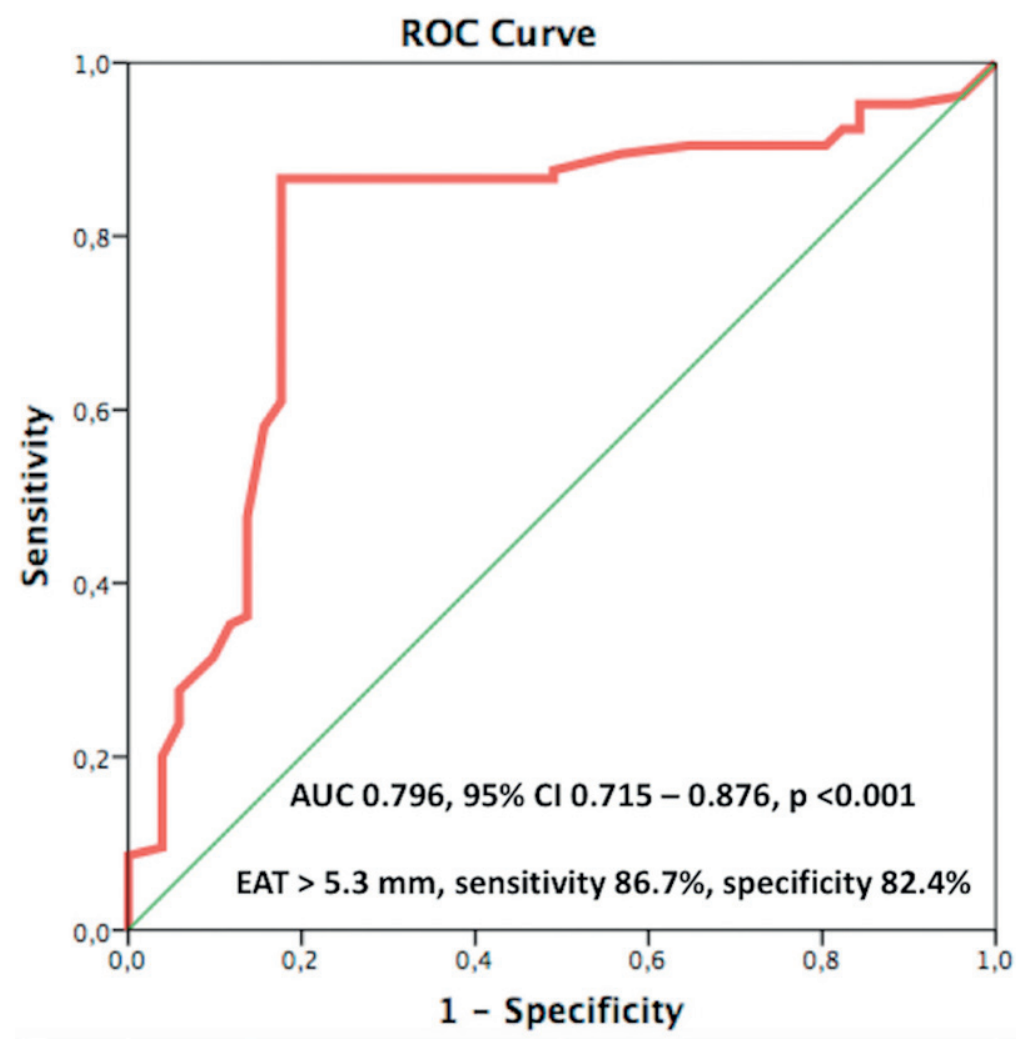

Fig. 2. The ROC curve analysis in prediction of high thrombus burden for EAT values.

vessel occlusion) as previously described ${ }^{10}$. The patients were grouped into 2 categories of thrombus grade as low thrombus burden (grades 0-3) and high thrombus burden (grades 4 and 5).

\section{Statistical analysis}

Statistical analysis were performed using the statistical package SPSS for Windows (Version 20.0; SPSS, Chicago, IL). Distribution of data was assessed by using one-sample Kolmogorov-Smirnov test. Values displaying normal distribution were expressed as mean $\pm \mathrm{SD}$, whereas values displaying non-normal distribution were expressed as median with interquartile range. Categorical variables were expressed as percentages. In case of no significant deviation from normality, significance of differences between 2 groups was tested with Student's ttest, otherwise Mann-Whitney U-test was used. Logistic regression analysis (univariate and multivariate) was used to assess the independent predictors of thrombus burden. White blood cell(WBC), EAT and waist circumference were used as independent covariates in the model. A receiver-operating characteristic (ROC) curve analysis was performed to determine the cutoff value of EAT to predict the presence of high thrombus burden (grade 4 and 5). A significance level was set at $P<0.05$.

\section{RESULTS}

A total of 156 patients were included in this study (51 in the low thrombus burden group and 105 in the high thrombus burden group). Baseline clinical, demographic features and laboratory findings of the two groups are shown in Table 1. There were no differences in the two groups for LVEF, smoking, family history of CAD, DM, HT, and hypercholesterolemia and for total cholesterol, triglyceride, GFR, LDL-C and HDL-C. Also, no significant differences were observed in gate-balloon time, painballoon time and culprit artery lesion locations between the 2 study groups.

WBC, fasting glucose level, EAT and waist circumference were significantly higher in the high thrombus burden group compared to the low thrombus burden group (Fig. 1).

The predictors of high thrombus burden were studied by multivariate logistic regression analysis. The EAT (odds ratio: $2.53,95 \% \mathrm{CI}$ : $1.76-3.67 ; P<0.001$ ) was found as an independent predictor of high thrombus burden (Table 2).

In the receiver operating characteristic(ROC) curve analysis, the thickness of $5.3 \mathrm{~mm}$ for EAT has $86.7 \%$ sensitivity and $82.4 \%$ specificity (AUC:0.796 95\% confidence intervals: $0.715-0.876 ; P<0.001$ ) (Fig. 2).

\section{DISCUSSION}

The present study results showed that increased EAT was an independent predictor of high thrombus burden in STEMI undergoing pPCI. To the best of our knowledge, this might be the first study investigating the relationship between EAT and coronary thrombus burden in STEMI. 
Table 1. Baseline clinical and angiographic charactersitics between patients with low vs high thrombus burden.

\begin{tabular}{|c|c|c|c|}
\hline & $\begin{array}{l}\text { Low thrombus burden } \\
\qquad \mathrm{n}=51\end{array}$ & $\begin{array}{l}\text { High thrombus burden } \\
\qquad \mathrm{n}=105\end{array}$ & $P$ \\
\hline Age, years & $53.7 \pm 11.2$ & $56.3 \pm 10.6$ & 0.730 \\
\hline Sex, male n (\%) & $43(85.5)$ & $87(82.9)$ & 0.657 \\
\hline Dislipidemia \% & $15(31.5)$ & $32(30.30)$ & 0.350 \\
\hline Familial History CAD, \% & $12(23)$ & $32(30.50)$ & 0.367 \\
\hline Waist circumference, $\mathrm{cm}$ & $95.37 \pm 11.22$ & $99.30 \pm 11.28$ & 0.046 \\
\hline BMI, $\mathrm{kg} / \mathrm{m}^{2}$ & $27.67 \pm 3.23$ & $27.99 \pm 3.9$ & 0.525 \\
\hline Killip class>II, n (\%) & $12.0(23.5)$ & $27.0(25.7)$ & 0.768 \\
\hline Fasting glucose level, mg/dL & $130.73 \pm 42.26$ & $142.29 \pm 48.13$ & 0.038 \\
\hline WBC, $\times 10^{9}$ & $11339 \pm 1997$ & $12656 \pm 3199$ & 0.001 \\
\hline HDL cholesterol, mg/dL & $39.5 \pm 9.87$ & $38.5 \pm 9.36$ & 0.454 \\
\hline LDL cholesterol, mg/dL & $135.2 \pm 29.2$ & $130.58 \pm 34.6$ & 0.334 \\
\hline $\mathrm{TG}, \mathrm{mg} / \mathrm{dL}$ & $131.0(103-184)$ & $136.0(85-195)$ & 0.962 \\
\hline GFR & $85.6 \pm 26.1$ & $94.4 \pm 34.6$ & 0.951 \\
\hline Fasting CKMB ng/mL & $34.0(24-55)$ & $44.0(25-57)$ & 0.193 \\
\hline Fasting Troponin $\mathrm{ng} / \mathrm{mL}$ & $4.40(2.0-8.0)$ & $5.0(3-7)$ & 0.398 \\
\hline LVEF, \% & $47.9 \pm 6.87$ & $46.9 \pm 7.09$ & 0.242 \\
\hline EAT thickness, mm & $4.76 \pm 1.08$ & $5.80 \pm 1.45$ & $<0.001$ \\
\hline Gate-baloon, $\min$ & $31.06 \pm 7.20$ & $31.88 \pm 7.43$ & 0.506 \\
\hline Pain-baloon, min & $140.0(90-189)$ & $152.0(97-249)$ & 0.192 \\
\hline Culprit lesion location & & & 0.698 \\
\hline LAD n, (\%) & $23(45.1)$ & $48(45.7)$ & \\
\hline CX n, (\%) & $16(31.4)$ & $25(23.8)$ & \\
\hline RCA n, (\%) & $12(27.6)$ & $31(29.5)$ & \\
\hline LMCA n, (\%) & $0(0.00)$ & $1(0.90)$ & \\
\hline Tirofiban $(+), \mathrm{n}(\%)$ & $27(52.9)$ & $73(69.5)$ & 0.051 \\
\hline Predilatation $(+), \mathrm{n}(\%)$ & $36(70.5)$ & $85(80.9)$ & 0.157 \\
\hline Stent length, mm & $21.81 \pm 8.60$ & $22.63 \pm 7.78$ & 0.487 \\
\hline Stent diameter, $\mathrm{mm}$ & $3.37 \pm 0.52$ & $3.37 \pm 0.47$ & 0.808 \\
\hline
\end{tabular}

Table 2. Logistic regression analysis in prediction of patients with high thrombus burden.

\begin{tabular}{lcccc}
\hline & Univariate OR, 95\% CI & $P$ & Multivariate OR, 95\% CI & $P$ \\
\hline WBC & $1.00(1.00-1.00)$ & 0.013 & $1.00(1.00-1.00)$ & 0.134 \\
EAT & $2.53(1.76-3.67)$ & $<0.001$ & $2.32(1.60-3.36)$ & $<0.001$ \\
Waist circumference, cm & $1.03(1.01-1.06)$ & 0.045 & $1.01(0.98-1.05)$ & 0.425 \\
\hline
\end{tabular}

Epicardial adipose tissue is related with various genes associated with extracellular matrix remodeling, inflammation, infection, and thrombosis pathways ${ }^{11}$. Inflammatory cells play a major role both in plaque disruption through the matrix degrading metalloproteinases and in triggering thrombosis following plaque disruption through the tissue factor pathway ${ }^{12}$. During that process, tumor necrosis factor $\alpha$ (TNF- $\alpha)$, monocyte chemoattractant protein 1 , interleukin $1 \beta$ (IL-1 $\beta$ ), interleukin 6 (IL-6), nerve growth factor, leptin, resistin, plasminogen activator inhibitor 1, and angiotensinogen especially WBC and many other pro-inflamatuar or anti-enflamatur cytokines have crucial roles within this cascade ${ }^{13,14}$. Y1lmaz et al. reported that lymphocyte and neutrophil counts, in particular the neutrophil/ lymphocyte ratio (NLR) could be used as independent predictors of thrombus burden in ACS (ref. ${ }^{15}$ ). In ACS, increased neutrophils are associated with the formation of platelet-leukocyte aggregates in the vascular lumen, which causes extension of the infarct area ${ }^{16}$. Neutrophils can also regulate platelet function by direct adhesion and paracrine effects of secreted factors. Recent studies revealed that, coronary thrombus burden is essentially important not only for the extension of the infarct area but also for the management prior to or during the coronary interventional procedures. Celik et al. reported the contributions of CRP and inflammatory markers on microvascular injury in STEMI (ref. ${ }^{17}$ ). High inflammatory markers may also be associated with adverse clinical outcomes in patients with ACS (ref. ${ }^{18-20}$ ). A composite marker of inflammation reflecting high trombus burden may provide additive information in the assessment of cardiovascular risk ${ }^{21}$. In our study, a statistically significant correlation was observed between WBC count and thrombus burden that might stem from very early PCI while acute phase reactors have not reached their peak serum levels. 
Body fat distribution, especially abdominal fat tissue, is more strongly correlated to epicardial fat and biochemical proprieties of EAT suggest its possible role as a cardiovascular and metabolic risk indicator. EAT was associated with metabolic syndrome and a significant correlation between metabolic risk factors such as blood pressure, fasting glucose, insulin resistance, and hyperlipidemia has been documented ${ }^{22}$. Epicardial, mesenteric, and omental fat all originate from the splanchnopleuric mesoderm which is a well-known pro-inflammatory source ${ }^{9,22}$. EAT releases both adiponectin and $\mathrm{TNF} \alpha$, and these two adipokines play important roles in heart diseases such as coronary artery disease ${ }^{23}$. Proinflammatory cytokines like IL-6, IL-1 $\beta$, TNF- $\alpha$ stimulate the production of tissue factor in monocytes to increase platelet reactivity and to trigger the production of plasminogen activator inhibitor 1 in the liver. Moreover, IL- 6 and TNF- $\alpha$ increase the risk of thrombosis by stimulating von Willebrand multimer secretion and another proinflammatory cytokines secreted from EAT, decreases the endothelial cell thrombomodulin expression and promotes thrombus formation ${ }^{24}$. Due to these facts, EAT is thought to be related to the presence and the severity of CAD, coronary plaque morphology and plaque rupture, myocardial ischemia, and major adverse cardiac events (MACE) in patient with ACS. In a recent study, increased EAT thickness were independent predictors of coronary thrombus formation in patients with NSTEMI who underwent pPCI and also coronary thrombus formation was independently associated with no-reflow phenomenon ${ }^{12}$. In compatible with those studies, the present study has shown that EAT was an independent predictor of coronary thrombus burden in STEMI who underwent pPCI.

\section{Study limitations}

The major limitation of this study was the small sample size. Cardiac magnetic resonance or computed tomography rather than echocardiography may give a more accurate assessment of epicardial fat amount. Adipokines and cytokines were not measured, and the exact biochemical relationship between EAT and thrombus burden was not precisely demonstrated. A prospective study is needed to determine the predictive value of EAT with high patient population. Since there was no data for predication for all of the subjects included to the study, the researchers didn't give information about the premedication of the patients. Lastly, in the present study only one blinded echocardiographer did the analysis. However, for the sake of interobserver variability there should be at least 2 independent blinded echocardiographers for the analysis.

\section{CONCLUSION}

EAT was an independent predictor of coronary thrombus burden in STEMI. Whether measuring the EAT preceding the coronary intervention may have clinical value in terms of reducing the adverse cardiac event at follow up post-PCI should be investigated in a prospectively designed trials.
Author contributions: AU, AK, SK, MD, ET: study design and drafting the manuscript; MD,ET: statistical analysis; AU, CD, MS, SC, UA: literature search: AU, AK, SK, MD, ET, CD, MS, SC, UA: data collection and management; AU, AK, SK, MD, ET, CD, MS, SC, UA: revision of the manuscript; All authors: approval of the latest version of the manuscript.

Conflict of interest statement: The authors state that there are no conflicts of interest regarding the publication of this article.

\section{REFERENCES}

1. Carr MC, Brunzell JD. Abdominal obesity and dyslipidemia in the metabolic syndrome: importance of type 2 diabetes and familial combined hyperlipidemia in coronary artery disease risk. J Clin Endocrinol Metab 2004;89(6):2601-7.

2. Comert N, Yucel O, Ege MR, Yaylak B, Erdogan G, Yilmaz MB. Echocardiographic epicardial adipose tissue predicts subclinical atherosclerosis: epicardial adipose tissue and atherosclerosis. Angiology 2012;63(8):586-90.

3. Greif M, Becker A, von Ziegler F, Lebherz C, Lehrke M, BroedI UC, Tittus J, Parhofer K, Becker C, Reiser M, Knez A, Leber AW. Pericardial adipose tissue determined by dual source $\mathrm{CT}$ is a risk factor for coronary atherosclerosis. Arterioscler Thromb Vasc Biol 2009;29(5):781-6.

4. Hirata Y, Kurobe H, Akaike M, Chikugo F, Hori T, Bando Y, Nishio C, Higashida M, Nakaya Y, Kitagawa T, Sata M. Enhanced inflammation in epicardial fat in patients with coronary artery disease. Int Heart J 2011;52(3):139-42.

5. Turak O, Ozcan F, Canpolat U, Isleyen A, Cebeci M, Oksuz F, Mendi MA, Cagli K, Golbasi Z, Aydogdu S. Increased echocardiographic epicardial fat thickness and high-sensitivity CRP level indicate diastolic dysfunction in patients with newly diagnosed essential hypertension. Blood Press Monit 2013;18(5):259-64.

6. Barron HV, Cannon CP, Murphy SA, Braunwald E, Gibson CM. Association between white blood cell count, epicardial blood flow, myocardial perfusion, and clinical outcomes in the setting of acute myocardial infarction: a thrombolysis in myocardial infarction 10 substudy. Circulation 2000;102(19):2329-34.

7. Cheitlin MD, Alpert JS, Armstrong WF, Aurigemma GP, Beller GA Bierman FZ, Davidson TW, Davis JL, Douglas PS, Gillam LD. ACC/ AHA Guidelines for the Clinical Application of Echocardiography. A report of the American College of Cardiology/American Heart Association Task Force on Practice Guidelines (Committee on Clinical Application of Echocardiography). Developed in collaboration with the American Society of Echocardiography. Circulation 1997;95(6):1686-744.

8. Schiller NB, Shah PM, Crawford M, DeMaria A, Devereux R, Feigenbaum H, Gutgesell H, Reichek N, Sahn D, Schnittger I, et al. Recommendations for quantitation of the left ventricle by two-dimensional echocardiography. American Society of Echocardiography Committee on Standards, Subcommittee on Quantitation of Two-Dimensional Echocardiograms. J Am Soc Echocardiogr 1989;2(5):358-67.

9. lacobellis G, Willens HJ. Echocardiographic epicardial fat: a review of research and clinical applications. J Am Soc Echocardiogr 2009;22(12):1311-9; quiz 1417-8.

10. lacobellis G, Barbaro G. The double role of epicardial adipose tissue as pro- and anti-inflammatory organ. Horm Metab Res 2008;40(7):442-5.

11. Gaborit B, Venteclef N, Ancel P, Pelloux V, Gariboldi V, Leprince P, Amour J, Hatem SN, Jouve E, Dutour A, Clement K. Human epicardial adipose tissue has a specific transcriptomic signature depending on its anatomical peri-atrial, peri-ventricular, or peri-coronary location. Cardiol Res 2015;108(1):62-73.

12. Bakirci EM, Degirmenci $H$, Duman $H$, Inci $S$, Hamur $H$, Buyuklu M, Ceyhun G, Topal E. Increased Epicardial Adipose Tissue Thickness is Associated With Angiographic Thrombus Burden in the Patients With 
Non-ST-Segment Elevation Myocardial Infarction. Clin Appl Thromb Hemost 2015;21(7):612-8.

13. Joseph L, Fink LM, Hauer-Jensen M. Cytokines in coagulation and thrombosis: a preclinical and clinical review. Blood Coagul Fibrinolysis 2002;13(2):105-16.

14. Nelson MR, Mookadam F, Thota V, Emani U, Al Harthi M, Lester SJ, Cha S, Stepanek J, Hurst RT. Epicardial fat: an additional measurement for subclinical atherosclerosis and cardiovascular risk stratification? J Am Soc Echocardiogr 2011;24(3):339-45.

15. Yilmaz M, Tenekecioglu E, Arslan B, Bekler A, Ozluk OA, Karaagac K, Agca FV, Peker T, Akgumus A. White Blood Cell Subtypes and Neutrophil-Lymphocyte Ratio in Prediction of Coronary Thrombus Formation in Non-ST-Segment Elevated Acute Coronary Syndrome. Clin Appl Thromb Hemost 2015;21(5):446-52.

16. Andrews RK, Berndt MC. Platelet physiology and thrombosis. Thromb Res 2004;114(5-6):447-53.

17. Celik T, lyisoy A, Yuksel UC, Jata B, Ozkan M. The impact of admission $\mathrm{C}$-reactive protein levels on the development of no-reflow phenomenon after primary $\mathrm{PCl}$ in patients with acute myocardial infarction: the role of inflammation. Int J Cardiol 2009;136(1):86-8; author reply 88-9.

18. Nunez J, Nunez E, Bodi V, Sanchis J, Minana G, Mainar L, Santas E, Merlos P, Rumiz E, Darmofal H, Heatta AM, Llacer A. Usefulness of the neutrophil to lymphocyte ratio in predicting long-term mortality in ST segment elevation myocardial infarction. Am J Cardiol 2008;101(6):747-52.
19. Tamhane UU, Aneja S, Montgomery D, Rogers EK, Eagle KA, Gurm HS. Association between admission neutrophil to lymphocyte ratio and outcomes in patients with acute coronary syndrome. Am J Cardiol 2008:102(6):653-7.

20. Azab B, Zaher M, Weiserbs KF, Torbey E, Lacossiere K, Gaddam S, Gobunsuy R, Jadonath S, Baldari D, McCord D, Lafferty J. Usefulness of neutrophil to lymphocyte ratio in predicting short- and long-term mortality after non-ST-elevation myocardial infarction. Am J Cardiol 2010;106(4):470-6.

21. Mazzone A, De Servi S, Ricevuti G, Mazzucchelli I, Fossati G, Pasotti D, Bramucci E, Angoli L, Marsico F, Specchia G, et al. Increased expression of neutrophil and monocyte adhesion molecules in unstable coronary artery disease. Circulation 1993;88(2):358-63.

22. Iacobellis G, Ribaudo MC, Assael F, Vecci E, Tiberti C, Zappaterreno A Di Mario U, Leonetti F. Echocardiographic epicardial adipose tissue is related to anthropometric and clinical parameters of metabolic syndrome: a new indicator of cardiovascular risk. J Clin Endocrinol Metab 2003;88(11):5163-8.

23. Sawaguchi T, Nakajima T, Hasegawa T, Shibasaki I, Kaneda H, Obi S, Kuwata T, Sakuma M, Toyoda S, Ohni M, Abe S, Fukuda H, Inoue T. Serum adiponectin and TNFa concentrations are closely associated with epicardial adipose tissue fatty acid profiles in patients undergoing cardiovascular surgery. Int J Cardiol Heart Vasc 2017:18:86-95

24. Esmon CT. The impact of the inflammatory response on coagulation. Thromb Res 2004;114(5-6):321-7. 